\title{
Construction of a non-Newtonian fluid model based on the finite difference lattice Boltzmann method
}

\author{
S. Tajiri \& M. Tsutahara \\ Graduate School of Science and Technology, Kobe University, Japan
}

\begin{abstract}
A model of the lattice Boltzmann method for non-Newtonian fluids was constructed. The shear stress of purely viscous but non-elastic non-Newtonian fluid is a function of shear rate only. For the power-law model, only two constant parameters can cover shear-thinning and shear-thickening fluids. Two power-law models are introduced to the finite difference lattice Boltzmann method. One is a model in which the collision parameter (the relaxation time) is determined as a function of the shear rate, and then the viscosity changes point by point according to the shear rate. For the other model, the effect of the variable viscosity is introduced as an external force which is determined by the local shear rate. Two-dimensional channel flow between two parallel plates was calculated by using the above two models. Both models are shown to give satisfactory results. However, some discontinuity appears in the calculation by the former model that is due to instability of the calculation. For the latter model, smooth velocity distributions are always obtained. The shear rate is estimated by the second order and fourth order central finite difference scheme, but the accuracy of the velocity distribution is to first order. A model in which the normal stress can be introduced by the shear is being constructed in the same manner. The normal stress was given by introducing the single mode Giesekus constitutive model to the finite difference lattice Boltzmann method.

Keywords: finite difference lattice Boltzmann method (FDLBM), non-Newtonian fluids, power-law model, Giesekus model.
\end{abstract}




\section{Introduction}

The lattice Boltzmann method (LBM) is a computational tool to analyze a thermal viscous fluid [1-4]. In particular, calculations of the flow within complicated porous media and the multi-phase flow are effective [5]. Recently, some models of LBM for purely viscous but non-elastic non-Newtonian fluid are proposed. The flow between parallel plates and open cavity flow were analyzed by Gabbanelli et al. [6] with the constructed LB model for the non-Newtonian fluid, and the examination about the calculation accuracy of the model was performed. Sullivan et al. [7] analyzed the detailed behaviour of the nonNewtonian fluid in three-dimensional complicated porous media. Their LB models gave the viscosity locally by determining the collision parameter (the relaxation time). The collision parameter was determined according to the shear rate. However, the relaxation time has a direct relation to the calculation stability, and the stability of the calculation is not enough. Yoshino et al. [8] presented the LB model for a non-Newtonian fluid using the Lattice Kinetic Scheme (LKS), in order to improve the problem. They analyzed the Darcy law of a two-dimensional porous media, and obtained the appropriate result.

In this paper, the non-Newtonian model of the finite difference lattice Boltzmann method (FDLBM) is presented. The viscosity of the non-Newtonian fluid was given using the relaxation time and the external force. The channel flow between two parallel plates was calculated by using the constructed models. Normal stress plays an important role in the non-Newtonian viscoelastic fluid. The model which can generate the normal stress was also constructed in the same manner using the external force.

\section{Numerical method}

The original two-dimensional and three-dimensional isothermal models (the D2Q9 and D3Q15 model [9]) are presented briefly in section 2.1. The introduced non-Newtonian model is presented in section 2.2. The power-law model is introduced for the non-Newtonian purely viscous fluid. The power-law model has only two constant parameters, and can cover shear-thinning and shearthickening fluids [10]. The single mode Giesekus constitutive model is used for the viscoelastic fluid $[11,12]$. The Giesekus model is a popular choice for several flows [13, 14]. For example, it is known that the Giesekus model is useful for describing processing flows of polymer solutions.

\subsection{Finite difference lattice Boltzmann method (multi particle model)}

A discrete BGK equation for the FDLBM is written as follows with the distribution function $f_{i}^{k}(\mathbf{x}, t)$ having the particle velocity $c_{i}$

$$
\frac{\partial f_{i}^{k}}{\partial t}+c_{i} \nabla f_{i}^{k}=-\frac{1}{\phi}\left(f_{i}^{k}-f_{i}^{e q k}\right)
$$


where $\phi$ is the collision parameter (the relaxation time) and $f_{i}^{e q k}$ is the local equilibrium distribution function chosen to satisfy the Navier-Stokes equation (refer to [9]). Macroscopic variables on each lattice site are defined as

$$
\begin{aligned}
\rho^{k} & =\sum_{i, k} f_{i}^{k}=\sum_{i, k} f_{i}^{e q k} \\
\sum_{k} \rho^{k} \mathbf{u} & =\sum_{i, k} f_{i}^{k} \mathbf{c}_{i}=\sum_{i, k} f_{i}^{e q k} \mathbf{c}_{i}
\end{aligned}
$$

for, respectively, the density, the momentum. For the finite difference lattice Boltzmann method, the governing equation is discretized, so the corresponding calculation procedures are given as follows. The time integration is performed by the second-order Runge-Kutta method and the third-order upwind scheme is employed for space dispersion of $c_{i} \nabla f_{i}$.

\subsection{Non-Newtonian model}

\subsubsection{Power-law model}

The constitutive equation of the viscosity of the power-law model is given as

$$
\eta=\eta_{0}|\dot{\gamma}|^{n-1}
$$

where $\eta_{0}$ and $n$ are the parameters of the power-law fluid. $n=1$ corresponds to the Newtonian fluid in eqn. (4). Then, $\eta_{0}$ is the coefficient of the Newtonian viscosity. $n>1$ corresponds to the shear-thickening fluid. $n<1$ corresponds to the shear-thinning fluid which decreases the viscosity coefficient when the shear rate becomes larger. The shear rate $\dot{\gamma}$ has a relation to the symmetrical rate of strain tensor $D_{\alpha \beta}$ as follows

$$
\begin{gathered}
\dot{\gamma}=\sqrt{D_{\alpha \beta} D_{\alpha \beta}} \\
D_{\alpha \beta}=\frac{1}{2}\left(\frac{\partial u_{\beta}}{\partial x_{\alpha}}+\frac{\partial u_{\alpha}}{\partial x_{\beta}}\right)
\end{gathered}
$$

where the subscript $\alpha$ and $\beta$ represent the Descartes coordinates and follow the summation convention. The velocity gradient $\partial u_{\alpha} / \partial x_{\beta}$ was derived by using the second order central difference scheme. The power-law model is introduced to the collision parameter $\phi$ and the external force $F_{\alpha}^{\text {power-law }}$ as follows

$$
\phi=3 \eta(D 2 Q 9), \quad \phi=\frac{3}{2} \eta(D 3 Q 15)
$$




$$
F_{\alpha}^{\text {power-law }}=-\frac{\partial}{\partial x_{\beta}}\left(\eta_{1} \frac{\partial u_{\alpha}}{\partial x_{\beta}}\right)+\frac{\partial}{\partial x_{\beta}}\left(\eta \frac{\partial u_{\alpha}}{\partial x_{\beta}}\right)
$$

$\eta_{1}$ is the original viscosity as the Newtonian fluid in eqn. (8). The above force is introduced to the local equilibrium distribution function in eqn. (3) by replacing

$$
\sum_{k} \rho^{k} u_{\alpha} \rightarrow \sum_{k} \rho^{k}\left(u_{\alpha}+\phi F_{\alpha}\right)
$$

\subsubsection{Single mode Giesekus constitutive model}

Here the viscoelastic fluid model proposed by Giesekus is shown briefly. The constitutive equation is

$$
\tau_{\alpha \beta}+\lambda \tau_{\alpha \beta}^{\nabla}+\alpha \frac{\lambda}{\eta_{0}} \tau_{\alpha \chi} \tau_{\chi \beta}=2 \eta_{0} D_{\alpha \beta}
$$

where $\tau_{\alpha \beta}$ represents the stress tensor. The non-linear term $\tau_{\alpha \chi} \tau_{\chi \beta}$ is a key role in the Giesekus model. $\alpha$ is the mobility factor $(0 \leq \alpha \leq 1) . \lambda$ is the relaxation time of the viscoelastc fluid. $\tau_{\alpha \beta}^{\nabla}$ represents the upper convected derivative as

$$
\tau_{\alpha \beta}^{\nabla}=\frac{\partial \tau_{\alpha \beta}}{\partial t}+u_{\chi} \frac{\partial \tau_{i \alpha \beta}}{\partial x_{\chi}}-\frac{\partial u_{i}}{\partial x_{\chi}} \tau_{\chi \beta}-\tau_{\alpha \chi} \frac{\partial u_{\chi}}{\partial x_{\beta}}
$$

The above equations correspond to the Giesekus model with non-considering the infinitesimal viscosity in the shear rate $\dot{\gamma} \rightarrow 0$. The Giesekus stress is introduced to the external force in the same manner with section 2.2.1. The total stress $\sigma_{\alpha \beta}$ of the Giesekus fluid is $\sigma_{\alpha \beta}=-P \delta_{\alpha \beta}+\tau_{\alpha \beta}$. Hence, the external force can be written as follows:

$$
F_{\alpha}^{\text {giesekus }}=-\frac{\partial}{\partial x_{\beta}}\left(-P \delta_{\alpha \beta}+\eta_{1} \frac{\partial u_{\alpha}}{\partial x_{\beta}}\right)+\frac{\partial \sigma_{\alpha \beta}}{\partial x_{\beta}}
$$

\section{Result and discussion}

\subsection{Channel flow between two parallel plates}

The channel flow between two parallel plates was calculated by using the two introduced power-law models. The exact solution of normalized velocity 


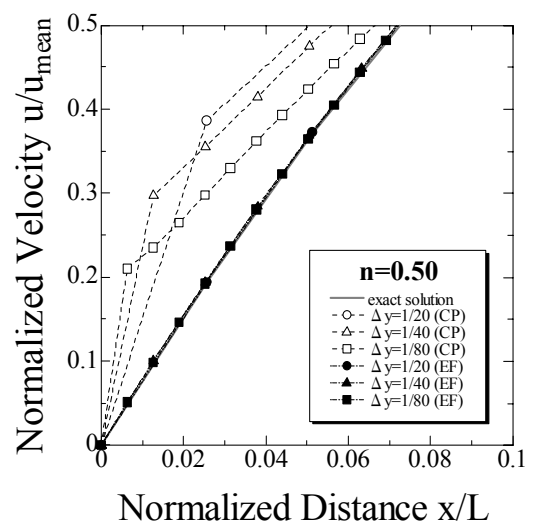

Figure 1: Normalized velocity profile near one of two parallel plates. The power-law exponent of the fluid is $n=0.50$ (shear thinning). Full symbols correspond to the external force (EF) model, and blank symbols correspond to the collision parameter (CP) model not giving the extrapolation of non-equilibrium distribution functions on the plate boundary.

$u_{\text {exa }}^{*}=u_{\text {exa }}(x) / u_{\text {exa_mean }}$ is written in eqn. (13). $u_{\text {exa_mean }}$ represents the mean velocity between two plates.

$$
u_{e x a}^{*}(x)=\frac{2 n+1}{n+1}\left[-\left(\frac{\left|\frac{L}{2}-x\right|}{\frac{L}{2}}\right)^{\frac{n+1}{n}}+1\right]
$$

where $\mathrm{L}$ represents the distance between two parallel plates. The external force model could give us the satisfactory velocity distribution, but the collision parameter model made a discontinuity of the velocity distribution near boundary in fig. 1 . The velocity discontinuity on boundary is the conventional characteristic of the FDLBM. This problem is improved by the below-mentioned technique. The distribution functions $f_{i}^{k}(\mathbf{x})$ on the plate boundary are given the extrapolation of non-equilibrium distribution functions as

$$
f_{i}^{k}(\mathbf{x})=f_{i}^{k e q}(\mathbf{x})+\left[2 f_{i}^{k n e q}(\mathbf{x}+\Delta \mathbf{x})-f_{i}^{k n e q}(\mathbf{x}+2 \Delta \mathbf{x})\right]
$$

The calculation result with the above technique agreed with the exact solution even if using the collision parameter model (fig.2). The evaluation about the external force model is described below. The satisfied velocity distribution was obtained in the parameter $n$ changed from 0.5 (shear-thinning fluid) to 1.5 (shear-thickening fluid) as shown in fig.3. 


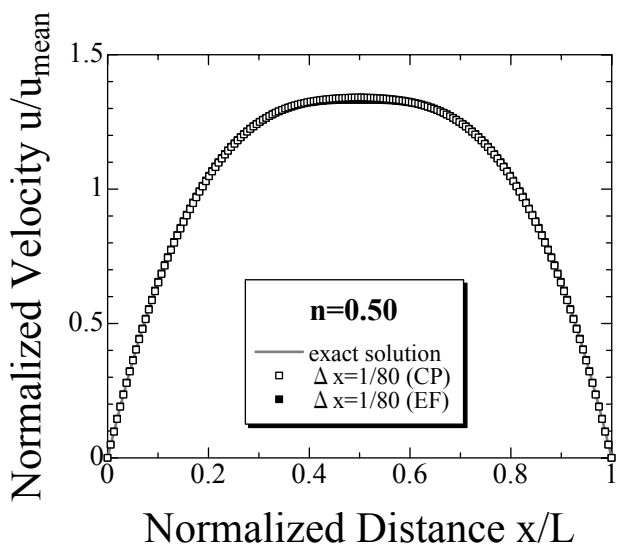

Figure 2: Normalized velocity profiles between two parallel plates. The power-law exponent of the fluid is $n=0.50$ (shear thinning). Full symbols correspond to the external force (EF) model, and blank symbols correspond to the collision parameter (CP) model. The distribution functions on the plate boundary are given the extrapolation of non-equilibrium distribution functions.

The accuracy of the velocity distribution was checked. The error norm in the steady flow (the velocity fluctuation become smaller than $10^{-8}$ ) was defined as

$$
\text { error }=\frac{\sum_{x}\left|u^{*}(x)-u_{\text {exa }}^{*}(x)\right|}{\sum_{x}\left|u_{\text {exa }}^{*}(x)\right|} \times 100[\%]
$$

where $u^{*}(x)$ is the velocity normalized by the mean velocity. The shear rate is estimated by the second order and also fourth order central finite difference scheme. The error norm in the highest resolution of lattice was about $0.02 \%$, but the accuracy of the velocity distribution is the first order as shown in fig. 4 .

\subsection{Shear flow of two component fluids on gravity field}

The shear flow with the free surface is simulated by using the introduced Giesekus model. The moderate diffusion scheme proposed by Latva-Kokko and Rothman [15, 16] was introduced to the FDLBM in order to simulate the behaviour of two immiscible phases. The governing equation (1) becomes

$$
\frac{\partial f_{i}^{k}}{\partial t}+c_{i} \nabla f_{i}^{k}=-\frac{1}{\phi}\left(f_{i}^{k}-f_{i}^{e q k}\right)+\left(f_{i}^{k}-f_{i}^{\prime k}\right)
$$




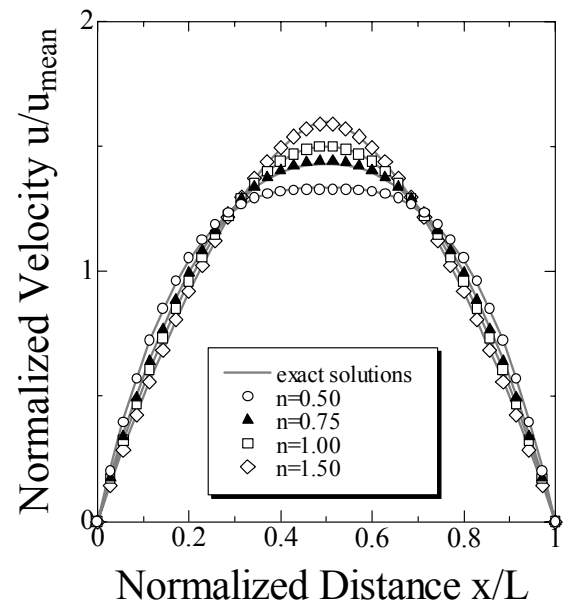

Figure 3: Normalized velocity profiles between parallel plates for different power indices by the external force model of the power-law fluid. The solid lines represent exact solutions.

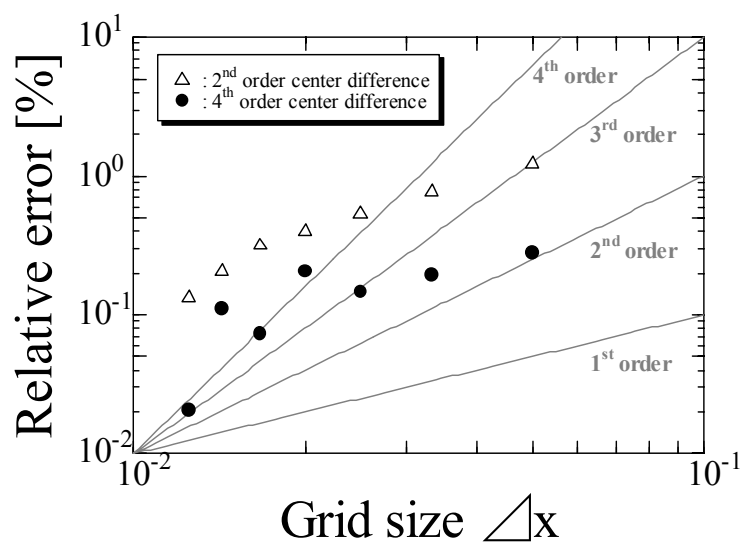

Figure 4: Error norms of the channel flow between parallel plates with $\Delta x=1 / 20,1 / 40,1 / 80$ by the external force model. $\Delta$ represent results of the $2^{\text {nd }}$ order central finite difference scheme for estimating the shear rate. $\bullet$ represent results of the $4^{\text {th }}$ order central finite difference scheme. The grey solid lines indicate inclines of the order. 
where $f_{i}^{\prime k}$ is the re- distribution function as follows:

$$
\begin{aligned}
& f_{i}^{\prime G}=\frac{\rho_{G}}{\rho_{G}+\rho_{N}}\left(f_{i}^{G}+f_{i}^{N}\right)+\left.\kappa \frac{\rho_{G} \rho_{N}}{\left(\rho_{G}+\rho_{N}\right)^{2}}\left(f_{i}^{e q G(0)}+f_{i}^{e q N(0)}\right) \cos \varphi\right|_{i} \\
& f_{i}^{\prime N}=\frac{\rho_{N}}{\rho_{G}+\rho_{N}}\left(f_{i}^{G}+f_{i}^{N}\right)-\left.\kappa \frac{\rho_{G} \rho_{N}}{\left(\rho_{G}+\rho_{N}\right)^{2}}\left(f_{i}^{e q G(0)}+f_{i}^{e q N(0)}\right) \cos \varphi\right|_{i}
\end{aligned}
$$

where subscript $\mathrm{N}$ and $\mathrm{G}$ represent the components of the fluid. The parameter $\kappa$ controls the thickness of interface. $f_{i}^{e q k(0)}$ is the zero-vector local equilibrium distribution function. $\varphi$ represents the angle between the particle direction and normal direction of the interface. The angle was determined as follows:

$$
\begin{gathered}
\left.\cos \varphi\right|_{i}=\frac{\mathbf{G} \cdot \mathbf{c}_{i}}{|\mathbf{G}| \cdot\left|\mathbf{c}_{i}\right|} \\
\mathbf{G}(\mathbf{x})=\sum_{i} \mathbf{c}_{i}\left[\rho^{G}\left(\mathbf{x}+\mathbf{c}_{i}\right)-\rho^{N}\left(\mathbf{x}+\mathbf{c}_{i}\right)\right]
\end{gathered}
$$

Fig. 5 shows the three-dimensional calculation domain. The calculation grid was $9 \times 31 \times 31$. The periodic boundary was employed in the $\mathrm{x}$-direction, and the other boundaries were non-slip condition. The right side boundary in fig. 5 moved at the velocity $\mathrm{U}_{0}=0.05$. Fig. $6($ a) is the pressure profile at the parameter $\alpha=1.0$ and $\lambda=10000$. It is shown that the pressure near a right-hand side boundary becomes large by the normal stress produced by the shear. Such pressure distribution in the Newtonian fluid is not seen (fig.6(b)). When the calculation time passed, the calculation became unstable.

\section{Conclusion}

The model of the finite difference lattice Boltzmann method for non-Newtonian fluids was presented. For purely viscous fluids, the power-law model is introduced to the collision parameter and the external force. Some discontinuity of velocity distributions appeared by using the collision parameter model in the two-dimensional channel flow between parallel plates. The stability of calculation was not enough. The external force model allowed us to obtain smooth velocity distributions. The single mode Giesekus constitutive model for viscoelastic fluids was also introduced in the same manner as the external force model. The normal stress appeared in the shear flow on the gravity field. However, the calculation was unstable. In the future work, the infinitesimal viscosity of the Giesekus model will have to be considered for improving the stability of calculation. 


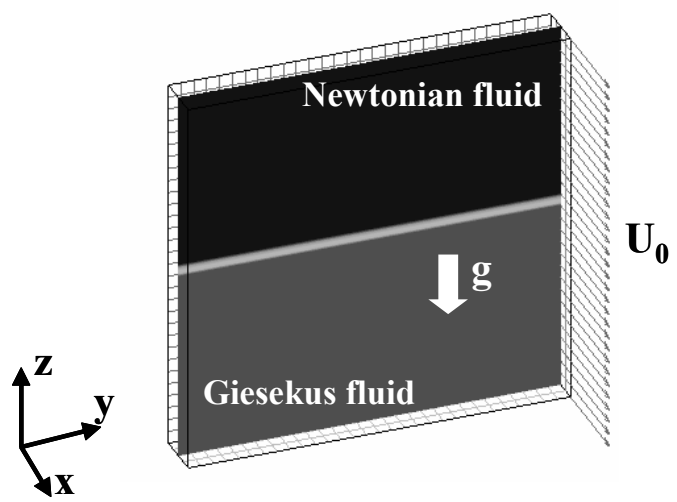

Figure 5: Calculation domain (grid number $9 \times 31 \times 31$ ). The boundary condition of $\mathrm{x}$-direction is the periodic. The other boundaries are non-slip solid walls. The velocity $\mathrm{U}_{0}=0.05$. The Giesekus fluid is given the gravity force $\mathrm{g}=0.0001$.

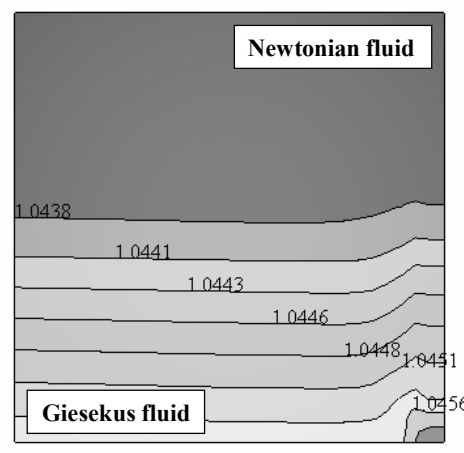

(a) Giesekus-Newtonian

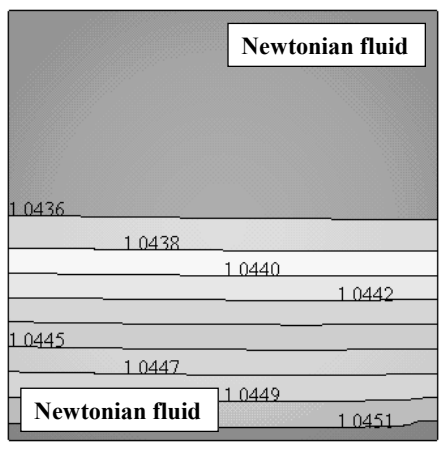

(b) Newtonian-Newtonian

Figure 6: Comparison of the pressure fields between the Giesekus fluid and the Newtonian fluid. The parameter $\alpha=1.0, \lambda=10000$.

\section{References}

[1] Qian, Y. H., Succi, S. \& Orszag, S. A., Recent Advances in Lattice Boltzmann Computing, Ann. Rev. of Comp. Phy. (D. Stauffer ed.), World Scientific, pp. 195-242, 1995.

[2] Rothman, D. H. \& Zalenski, S., Lattice-Gas Celluar Automata, Cambridge U. P., pp. 73-90, 1997.

[3] Chen, S. \& Doolen, G. D., Lattice Boltzmann Method for Fluid Flows, Ann. Rev. Fluid Mech., Ann. Rev. Inc, pp. 329-364, 1998. 
[4] S. Succi, The lattice Boltzmann Equation for Fluid Dynamics and Beyond, Oxford, pp. 51-123, 2001.

[5] Inamuro, T., Ogata, T., Tajima, S. \& Konishi, N., A lattice Boltzmann method for incompressible two-phase flows with large density differences, J. Computational Physics, 198, pp. 628-644, 2004

[6] Gabbanelli, S., Drazer, G. \& Koplik, J., Lattice Boltzmann Method for Non-Newtonian (Power-law) Fluids, Phys. Rev. E, 72, 2005.

[7] Sullivan, S. P., Gladden, L. F. \& Johns, M. L., Simulation of Power-Law Fluid Flow through Porous Media using Lattice Boltzmann Techniques, $J$. Non-Newton. Fluid Mech., 133, pp.91-98, 2006.

[8] Yoshino, M., Hotta, Y., Hirozane, T. \& Endo, M., A Lattice Boltzmann Method for Non-Newtonian Fluid Flows, Japan Society for Computational Methods in Engineering, 6(2), 2006.

[9] Tsutahara, M., Takada, N. \& Kataoka, T., Lattice Gas Method \& Lattice Boltzmann Method, Corona-sya, pp.101-112, 1999; in Japanese.

[10] Nakamura, K., Non-Newtonian Fluid Mechanics. Corona-sya, pp.68-71, 1997; in Japanese

[11] Giesekus, H., A Simple Constitutive Equation for Polymer Fluids Based on the Concept of Deformation-dependent Tensorial Mobility, J. NonNewton. Fluid Mech., 11, pp.69-109, 1982.

[12] Giesekus, H., Stressing behaviour in simple shear flow as predicted by a new constitutive model for polymer fluids, J. Non-Newton. Fluid Mech., 12, pp.376-374, 1983.

[13] Yoo, J. Y. \& Choi, H. Ch., On the steady simple shear flows of the onemode Giesekus fluid, Rheologica Acta, 28, pp.13-24, 1989.

[14] Mostafaiyan, M., Khodabandehlou, K. \& Sharif, F., Analysis of a viscoelastic fluid in an annulus using Giesekus model, J. Non-Newton. Fluid Mech., 118, pp.49-55, 2004.

[15] Latva-Kokko, M. \& Rothman, D. H., Diffusion properties of gradientbased lattice Boltzmann models of immiscible fluids, Phys. Rev. E, 71, 2005.

[16] Latva-Kokko, M. \& Rothman, D. H., Static contact angle in lattice Boltzmann models of immiscible fluids, Phys. Rev. E, 72, 2005. 